\title{
Intraarticular Entrapment of Os Subfibulare Following a Severe Inversion Injury of the Ankle: A Case Report
}

\author{
Ozkan Kose ${ }^{1, *}$; Omer Faruk Kilicaslan ${ }^{1}$; Ferhat Guler ${ }^{1}$; Cemil Aktan ${ }^{1}$ \\ ${ }^{1}$ Department of Orthopedics and Traumatology, Antalya Education and Research Hospital, Antalya, Turkey \\ ${ }^{*}$ Corresponding author: Ozkan Kose, Department of Orthopedics and Traumatology, Antalya Education and Research Hospital, Antalya, Turkey. Tel: +90-5326422612, Fax: \\ +90-2422494400, E-mail: drozkankose@hotmail.com \\ Received: January 17, 2015; Accepted: February 16, 2015
}

\begin{abstract}
Introduction: Anterior Talofibular Ligament (ATFL) rupture is the most commonly injured anatomic structure in lateral ankle sprain. In some cases, ATFL avulsion fracture from the lateral malleolus may occur instead of purely ligamentous injuries. The ATFL avulsion fracture is detected as a small ossicle at the tip of lateral malleolus on direct radiographs, which is called os subfibulare in chronic cases.

Case Presentation: Severe displacement of this ossicle to the tibiotalar joint space is an extremely rare injury. Herein, a case of intraarticular entrapment of os subfibulare following a severe inversion injury of the ankle, which caused a diagnostic challenge was presented. Conclusions: To the best of our knowledge, this is the first case of entrapment of os subfibulare in the talotibial joint space. Fixation of the os subfibulare to lateral malleolus resulted in union and excellent functional results.
\end{abstract}

Keywords: Anterior Talofibular Ligament; Os Subfibulare; Ankle Sprain

\section{Introduction}

Os subfibulare is a round to elongated shape accessory ossicle located under the tip of the lateral malleolus. Incidence of os subfibulare in radiographic survey studies has been reported between $0.2 \%$ and $2.1 \%$ in general population $(1,2)$. The origin of os subfibulare is not clearly understood, but there are two recognized theories regarding its origin. Some authors proposed that it is a true sesamoid bone formed by an accessory ossification center $(2,3)$. However, some authors suggested that os subfibulare is not a true sesamoid bone, but rather an old nonunited avulsion fracture of the lateral malleolus resulting from traction of the Anterior Talofibular Ligament (ATFL) $(4,5)$.

Ankle sprain is one of the most common musculoskeletal injuries presenting to emergency departments. Lateral ankle ligaments, particularly the ATFL, is the most commonly injured anatomic structure (6). Anterior talofibular ligament injuries can occur in three distinct forms; rupture of the ligament as a pure soft tissue injury, an avulsion fracture at the ATFL's attachment to the fibula or talus (7). In earlier studies, the rate of ATFL avulsion fracture in ankle sprain has been reported lower compared to recent studies. Brostrom et al. evaluated 205 injuries to the ATFL with arthrography and detected 28 (14\%) ATFL avulsion fractures (8). Haraguchi et al. described new radiographic projections to demonstrate the avulsion fracture of ATFL and prospectively evaluated 169 ankle sprains and detected 44 (26\%) ATFL avulsion fractures from the lateral malleolus. They claimed that the prevalence of ATFL avulsion fractures may be higher than previously thought $(9,10)$.
In acute lateral ankle sprain, if the ATFL avulsion fracture occurs, it may unite to the distal malleolus. If pseudoarthrosis or fibrous union develops, avulsed fragment remains as a separate ossicle. Haraguchi et al. reported osseous union in 24 (65\%) of 37 patients with avulsion fracture presenting with ankle sprain. Union was significantly influenced by the degree of displacement (10). Several previous studies showed a higher prevalence of os subfibulare in patients with chronic lateral ankle instability (11). However, it is hard to discriminate a real os subfibulare (formed by accessory ossification center) from an ATFL avulsion fracture in chronic cases.

In most cases the ATFL avulsion fracture or os subfibulare is located just anterior or inferior to the tip of lateral malleolus. Severe displacement of this ossicle to the tibiotalar joint space is an extremely rare injury. Herein, a case of intraarticular entrapment of os subfibulare following a severe inversion injury of the ankle, which caused a diagnostic challenge was presented. To the best of our knowledge, this is the first case of entrapment of os subfibulare in the talotibial joint space.

\section{Case Presentation}

A 19-year-old woman admitted to our emergency department immediately after she sustained an ankle sprain. On presentation she was unable to weight bear and her ankle was severely swollen and deformed. Active and passive ankle movements were painful and restricted. Neurovascular examination had normal findings. Her medical history revealed that she sustained several

Copyright (c) 2015, Kashan University of Medical Sciences. This is an open-access article distributed under the terms of the Creative Commons Attribution-NonCommercial 4.0 International License (http://creativecommons.org/licenses/by-nc/4.0/) which permits copy and redistribute the material just in noncommercial usages, provided the original work is properly cited. 
episodes of ankle sprain previously and had chronic lateral ankle pain and symptoms of lateral ankle instability. Plain radiographic examination of the ankle showed an intraarticular bone fragment in the tibiotalar joint that led to a significant talar tilt $\left(13^{\circ}\right)$ (Figure $1 \mathrm{~A}$ ) and (Figure $1 \mathrm{~B})$ Initially it was thought that this fragment originated from talus and most probably it was a large osteochondral fracture. Further CT examination demonstrated that this fragment was avulsed from the tip of lateral malleolus and there was no fracture in talus or plafond (Figure 1C) and (Figure 1 D). Therefore, a provisional diagnosis of intra-articular entrapment of ATFL avulsion fracture was made and surgical exploration was planned.

Under spinal anesthesia and tourniquet control, an oblique incision was made on the anterolateral aspect of the ankle. Retinaculum, lateral ligaments and the joint
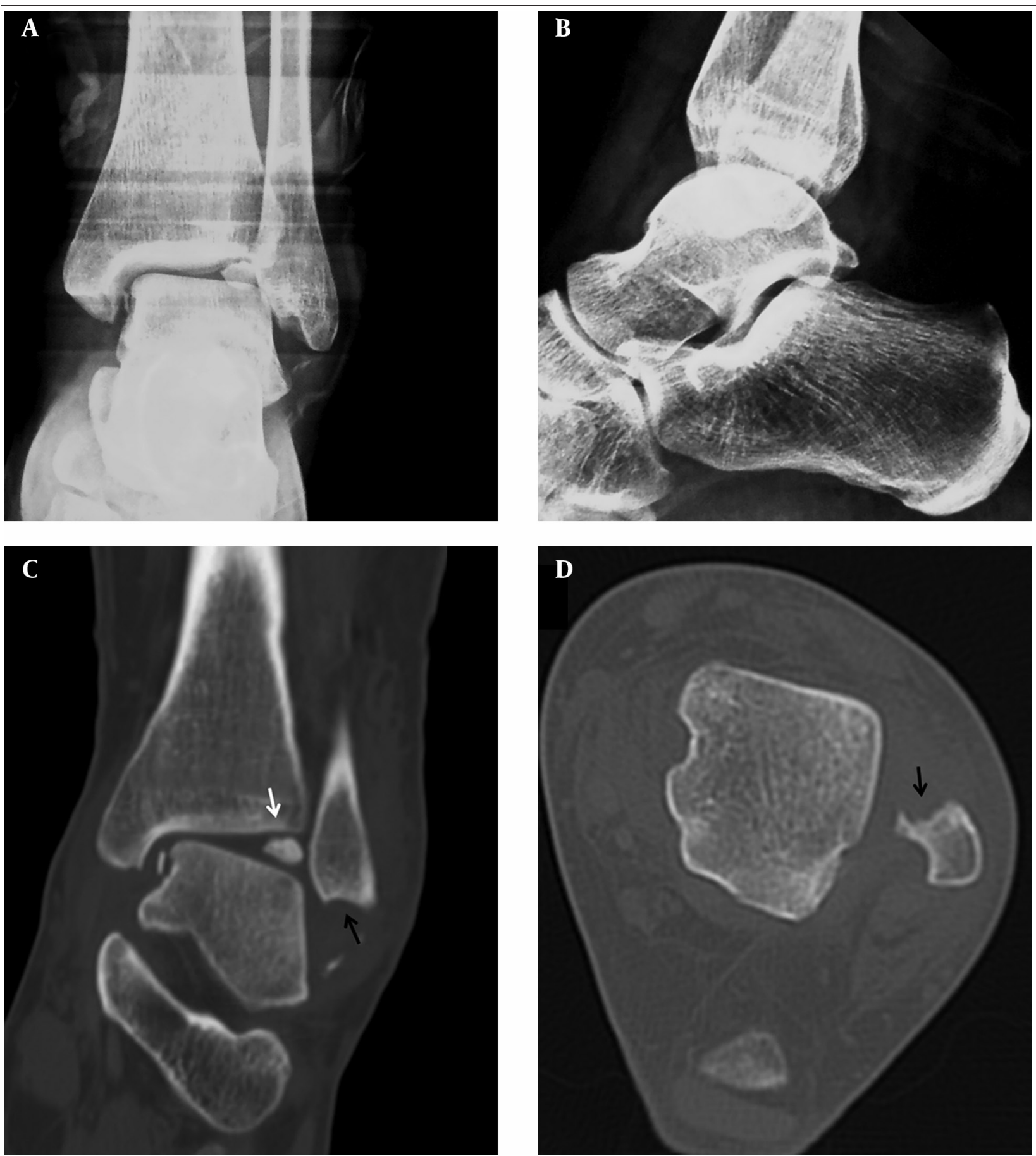

Figure 1. A, Anteroposterior; B, Lateral X-Ray at Initial Admission, A Large Fragment Is Seen in Tibiotalar Joint with Talar Tilt; C, Coronal; D, Axial Computed Tomography Image of the Ankle Demonstrates the Fragment (White Arrow), Note the bone defect at the tip of fibula (black arrows) 
capsule were dissected and exposed. During intraoperative examination, the fragment was found to be attached to the ATFL. The bone fragment was large, oval shaped and corticated. There was a bone defect at the tip of malleolus and the fragment was compatible with this bone defect. The distal tip of the fibula was also corticated. The joint capsule was repaired and the fragment was fixed with two cannulated headless compression screw after decortication of the fracture plane with curettage. A below knee cast was applied for patient for six weeks.

After six weeks, the cast was removed and a gradual physical therapy program involving ankle range of mo- tion and strengthening was initiated. At the final followup, nine months after the surgery, the patient was invited back to the hospital and final radiographic and clinical examination was performed. The patient was free of pain and returned back to her previous level of daily activity; the range of motion was normal compared with the contralateral side. There was no history of ankle sprain during the follow-up. The anterior drawer test had negative result. The American Orthopedic Foot and Ankle Society (AOFAS) score was 90. Final ankle radiographs showed union of the fracture and stress radiographs showed less than 5 degree of talar tilt compared to uninjured side (Figure 2).
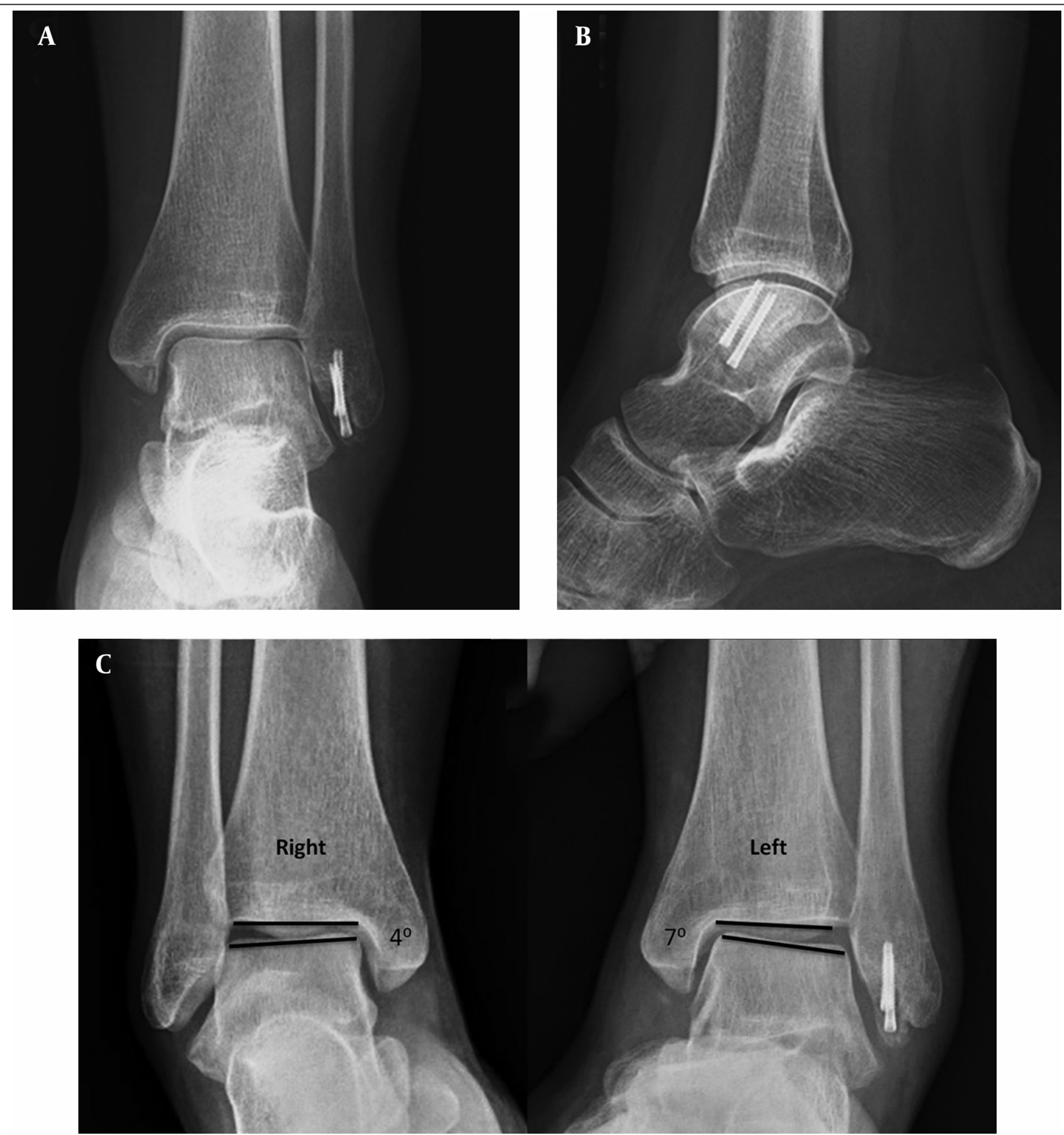

Figure 2. A, Anteroposterior; B, Lateral X-Ray at the Final Follow-up Showed Union; C, Stress Radiographs of Both Ankles Showed Nearly Normal Talar Tilt 


\section{Discussion}

Currently, initial management of acute ankle sprain is a conservative treatment in the form of rest, ice, elevation and compression followed by early functional bracing that prevents inversion of the ankle. This treatment strategy is usually effective to reestablish ankle stability and most patients heal without functional impairment and chronic symptoms (12). However, despite appropriate treatment, $10 \%$ to $30 \%$ of patients may have pain, residual instability and recurrent ankle sprains (13). In these patients, further conservative treatments composed of muscle strengthening, particularly the peroneal muscles, proprioceptive exercises and bracing may provide benefit and prevent recurrent ankle sprains. Failure of conservative treatment, pain and apparent ankle instability that interfere with daily activities and participation of sporting activities and findings of severe talar tilt on stress radiographs are current surgical indications for lateral ankle instability (12).

In cases presenting with os subfibulare and chronic ankle instability, the best treatment method is not wellestablished. Various treatment methods have been used up to date. Han et al. reported excellent results with simple arthroscopic excision of the os subfibulare without ligament reconstruction. They claimed that no further ankle instability occurred in their patients (11). However, Monden et al. proposed that arthroscopic ossicle excision without avoiding harm to the ATFL is impossible and ATFL reconstruction should be performed to reliably ensure proper ligament function after the excision (14). Pill et al. reported good and excellent results with ossicle removal and ATFL reconstruction in children with chronic pain and instability associated with os subfibulare (15). Kim et al. reported inferior functional outcomes in ankles with ossicles and suggested fusion of the ossicles when performing ligament reconstruction in chronic lateral ankle instability with ossicles over 10 $\mathrm{mm}$ (16). On the contrary, Chun et al. claimed that osteosynthesis of the ossicles may not be necessary, even if the size is considerable (17). In the presented case, ossicle was large enough to be fixed with screws and more importantly the AFTL was intact and attached to the fragment. We thought that bone to bone healing would be better than ligament to bone healing, thus performed the fixation of fragment. During the follow-up, the ossicle fused to the lateral malleolus and resulted in satisfactory functional result.

In conclusion, recurrent and further traumatic injuries may occur to the preexisting os subfibulare and cause detachment and even intraarticular entrapment. Direct radiograph may not delineate the origin of this fragment and CT should be the choice of imaging modality. If ATFL is firmly attached to this fragment and the fragment is large, fixation may be a good choice of treatment.

\section{Authors' Contributions}

Study concept and design: Ozkan Kose, Omer Faruk Kilicaslan and Ferhat Guler. Drafting of the manuscript: Ozkan Kose and Ferhat Guler. Critical revision of the manuscript for important intellectual content: Cemil Aktan and Ozkan Kose. Administrative, technical and material support: OFK. Study supervision: Ozkan Kose and Ferhat Guler.

\section{References}

1. Mellado JM, Ramos A, Salvado E, Camins A, Danus M, Sauri A. Accessory ossicles and sesamoid bones of the ankle and foot: imaging findings, clinical significance and differential diagnosis. Eur Radiol. 2003;13 Suppl 4:L164-77.

2. Champagne IM, Cook DL, Kestner SC, Pontisso JA, Siesel KJ. Os subfibulare. Investigation of an accessory bone. J Am Podiatr Med Assoc. 1999;89(10):520-4.

3. Kono T, Ochi M, Takao M, Naito K, Uchio Y, Oae K. Symptomatic os subfibulare caused by accessory ossification: a case report. Clin Orthop Relat Res. 2002(399):197-200.

4. Berg EE. The symptomatic os subfibulare. Avulsion fracture of the fibula associated with recurrent instability of the ankle. $J$ Bone Joint Surg Am. 1991;73(8):1251-4.

5. Ferran J, Blanc T. [Os subfibulare in children secondary to an osteochondral fracture]. J Radiol. 2001;82(5):577-9.

6. Doherty C, Delahunt E, Caulfield B, Hertel J, Ryan J, Bleakley C. The incidence and prevalence of ankle sprain injury: a systematic review and meta-analysis of prospective epidemiological studies. Sports Med. 2014;44(1):123-40.

7. Miyamoto W, Takao M, Nishiguchi K, Uchio Y. Technique tip: a radiographic projection for an avulsion fracture of the talar attachment of the anterior talofibular ligament. Foot Ankle Int. 2008;29(4):435-7.

8. Brostrom L. Sprained ankles. 3. Clinical observations in recent ligament ruptures. Acta Chir Scand.1965;130(6):560-9.

9. Haraguchi N, Kato F, Hayashi $\mathrm{H}$. New radiographic projection for avulsion fractures of the lateral malleolus.J Bone Joint Surg Br. 1998;80(4):684-8.

10. Haraguchi N, Toga H, Shiba N, Kato F. Avulsion fracture of the lateral ankle ligament complex in severe inversion injury: incidence and clinical outcome. Am J Sports Med. 2007;35(7):1144-52.

11. Han SH, Choi WJ, Kim S, Kim SJ, Lee JW. Ossicles associated with chronic pain around the malleoli of the ankle. J Bone Joint Surg Br. 2008;90(8):1049-54.

12. Colville MR. Surgical treatment of the unstable ankle. J Am Acad Orthop Surg. 1998;6(6):368-77.

13. Lofvenberg R, Karrholm J, Lund B. The outcome of nonoperated patients with chronic lateral instability of the ankle: a 20-year follow-up study. Foot Ankle Int. 1994;15(4):165-9.

14. Monden S, Hasegawa A, Hio N, Taki M, Noguchi H. Arthroscopic excision of separated ossicles of the lateral malleolus. J Orthop Sci. 2013;18(5):733-9.

15. Pill SG, Hatch M, Linton JM, Davidson RS. Chronic symptomatic os subfibulare in children. J Bone Joint Surg Am. 2013;95(16):e115(1-6).

16. Kim BS, Choi WJ, Kim YS, Lee JW. The effect of an ossicle of the lateral malleolus on ligament reconstruction of chronic lateral ankle instability. Foot Ankle Int. 2010;31(3):191-6.

17. Chun TH, Park YS, Sung KS. The effect of ossicle resection in the lateral ligament repair for treatment of chronic lateral ankle instability. Foot Ankle Int. 2013;34(8):1128-33. 